\title{
Cultivation of circulating tumor cells in esophageal cancer
}

\author{
Vladimir Bobek ${ }^{1-3}$, Rafal Matkowski ${ }^{4}$, Robert Gürlich ${ }^{5}$, Krzysztof Grabowski, \\ Jolanta Szelachowska ${ }^{7}$, Robert Lischke ${ }^{3}$, Jan Schützner ${ }^{3}$, Tomas Harustiak ${ }^{3}$, \\ Alexandr Pazdro ${ }^{3}$, Adam Rzechonek ${ }^{2}$, Katarina Kolostova ${ }^{1}$
}

${ }^{1}$ Department of Laboratory Genetics, University Hospital Kralovske Vinohrady, Prague, Czech Republic

${ }^{2}$ Department of Histology and Embryology, Wroclaw Medical University, Wroclaw, Poland

${ }^{3} 3^{\text {rd }}$ Department of Surgery, $1^{\text {st }}$ Faculty of Medicine Charles University in Prague and University Hospital Motol, Prague, Czech Republic

${ }^{4}$ Division of Oncological Surgery and Department of Oncology, Lower Silesian Oncology Centre, Wroclaw Medical University, Wroclaw, Poland

${ }_{5}^{5}$ Department of Surgery, $3^{\text {rd }}$ Faculty of Medicine Charles University Prague and University Hospital Kralovske Vinohrady, Prague, Czech Republic

${ }^{6}$ Department of Gastrointestinal and General Surgery, Wroclaw Medical University, Wroclaw, Poland ${ }^{7}$ Department of Oncology, Wroclaw Medical University, Lower Silesian Oncology Centre, Wroclaw, Poland

\begin{abstract}
The presence of circulating tumor cells (CTCs) in patients with metastatic carcinoma is generally associated with poor clinical outcome. There have been many investigations showing a possible use of CTCs as minimally invasive predictive and prognostic biomarker in cancer medicine. In this report a size-based method $\left(\mathrm{MetaCell}^{\circledR}\right)$ for quick and easy enrichment and cultivation of CTCs is presented to enable possible CTCs use in esophageal cancer (EC) management. In total, 43 patients with diagnosed EC, 20 with adenocarcinoma (AdenoCa) and 23 with squamous cell carcinoma (SCC), were enrolled into the adaptive prospective-like study. All the patients were candidates for surgery. The CTCs were detected in 27 patients (62.8\%), with a higher rate in adenocarcinoma (75\%) than SCC (52\%). Finally, there were 26 patients with resectable tumors exhibiting CTCs-positivity in $69.2 \%$ and 17 patients with non-resectable tumors with $41.7 \%$ CTCs-positivity. Interestingly, in the patients undergoing neoadjuvant therapy, the CTCs were detected at time of surgery in $55.5 \%(10 / 18)$. The overall size-based filtration approach enabled to isolate viable CTCs and evaluate to their cytomorphological features by means of vital fluorescent staining. The CTCs were cultured in vitro for further downstream applications including immunohistochemical analysis. This is the first report of the successful culturing of esophageal cancer CTCs. The detection of CTCs presence could help in the future to guide timing of surgical treatment in EC patients. (Folia Histochemica et Cytobiologica 2014, Vol. 52, No. 3, 171-177)
\end{abstract}

Key words: esophageal cancer; circulating tumor cells; MetaCell ${ }^{\circledast}$; CTCs culture; gastrointestinal tumors

Correspondence address: V. Bobek M.D., Ph.D.

University Hospital Kralovske Vinohrady

Department of Laboratory Genetics

Srobarova St. 50, 10034 Prague, Czech Republic

tel.: +420 267163578

e-mail:vbobek@centrum.cz

\section{Introduction}

Esophageal cancer (EC) is currently the most rapidly increasing cancer in the U.S. and Western Europe [1]. EC is the eighth most common cancer and the sixth most common cause of cancer death worldwide, affecting more male than female [2]. The survival and prognosis of EC patients depend on the stage 
of the tumor at the time of detection. Early tumor diagnosis, improved surgical treatment and multimodal therapeutic concepts have helped to reduce the mortality of EC patients. Nevertheless, the survival rates of these patients are still highly unsatisfactory. The prognosis depends on local tumor recurrence and/or the occurrence of distant metastases. Esophageal cancer is notorious for its aggressive biological behavior, local infiltration, involvement of adjacent lymph nodes, and metastasis through hematogenous spread. It has been reported that the frequency of hematogenous recurrence was high despite radical surgery with lymph node dissection [3]. In this regard, the detection of cancer cells in the blood could be important in identifying patients with high relapse risk. The presence of CTCs in patients with metastatic carcinoma is generally associated with poor clinical outcome [4]. This has been shown for both malignancies in the gastrointestinal tract $[5,6]$ and other cancers $[7,8]$.

Many investigations showed a positive correlation between detection of circulating tumor cells (CTCs), tumor staging and patient prognosis. Therefore, in general, CTCs are thought to provide additional prognostic information for the TNM classification. Similarly, it is hoped that CTCs detection could help to guide the surgery timing in EC patients, especially in the group of EC patients undergoing neoadjuvant chemotherapy. We report a new method for quick and easy enrichment and cultivation of CTCs isolated from the EC patients' blood.

\section{Material and methods}

Patients. To date, 43 patients with diagnosed EC, 20 adenocarcinomas (AdenoCa) and 23 squamous cell carcinomas (SCC), localized in esophagus or stomach maximally $5 \mathrm{~cm}$ from gastro-esophageal junction, were enrolled into the study in accordance with the Declaration of Helsinki. All patients were candidates for surgery treatment. Based on the informed consent, the clinical data were collected from all participating patients. The patient characteristics are shown in Table 1. Peripheral blood was collected prior to surgery. For each patient, approximately $8 \mathrm{~mL}$ of venous blood was drawn from the antecubital vein into S-Monovette tubes (Sarstedt AG \& Co., Numbrecht, Germany) containing $1.6 \mathrm{mg}$ EDTA $/ \mathrm{mL}$ blood as an anticoagulant. The samples were processed at room temperature using an isolation procedure completed within 24 hours after the blood draw. The biological material has been collected in a prospective time matter; however this was not a clinical study, but experimental one which aimed to test the feasibility of a new CTC isolation method.
CTCs enrichment and culture. Recently, a new size-based separation method for viable CTCs - enrichment from unclotted peripheral blood has been introduced (MetaCell ${ }^{\circledR}$, MetaCell s.r.o., Ostrava, Czech Republic). The size-based enrichment process is based on the filtration of peripheral blood (PB) through porous polycarbonate membrane (pores with diameter of $8 \mu \mathrm{m}$ ). The minimum and maximum volume of the filtered $\mathrm{PB}$ may be adjusted up to $50 \mathrm{~mL}$ of fluid. Routinely, $8 \mathrm{~mL}$ PB from EC patients was transferred into the filtration tube. The PB flow through filter is supported by capillary action of the absorbent touching the membrane filter. The whole isolation procedure was performed at room temperature. The enriched CTCs were observed on the membrane immediately after filtration by inverted light microscopy and subsequently (after 2 hours) by fluorescent microscopy using unspecific vital nuclear stain (NucBlue $^{\mathrm{TM}}$, Life Technologies). For some of the tested samples unspecific cytoplasmic vital stain (CellTracker ${ }^{\mathrm{TM}}$, Life Technologies) was used to identify viable CTCs. The fluorescent analysis enables to distinguish cytomorphology of the recently enriched CTC fraction with a very high percentage of sensitivity. The presence of the isolated CTCs controlled immediately after the isolation process helped to avoid false negative results.

Afterwards, the membrane filter kept in a plastic ring was transferred into the 6-well culture plate, $4 \mathrm{~mL} \mathrm{RPMI}$ media was added to the filter top and CTCs were cultured under standard conditions on the membrane in vitro under conditions of standard cancer cell cultures $\left(5 \% \mathrm{CO}_{2}, 37^{\circ} \mathrm{C}\right)$ and observed by inverted microscope. The CTCs are grown in the $10 \%$ fetal bovine serum-enriched RPMI medium for the period of maximum 14 days. The cultured cells were stained by the May-Grünwald method and immunohistochemistry using the tumor-specific antibodies to prove the cell origin (antiCK18-FITC, Sigma-Aldrich, Steinheim, Germany) and to visualize cell nuclei (DAPI staining, Sigma-Aldrich).

Next, the enriched CTCs fraction was transferred from the membrane and cells were cultured directly on the plastic surface or microscopic slides. Microscopic slide culture is preferred if the immunohistochemistry/immunofluorescence analysis is planned. If intermediate CTCs-analysis is planned, the CTCs fraction is transferred in phosphate-buffered saline (1,5 mL PBS) to the cytospin slide. The slide was then dried for 24 hours and analyzed by means of immunohistochemistry.

Cytomorphological analysis. The fixed and stained cells on the membrane were examined using light and fluorescence microscopy. The analytical process can be divided into two steps: (1) observing at smaller magnification (up to $20 \times$ ) to identify cells or cell nuclei, (2) observing at higher magnification (up to $60 \times$ ) for more detailed evaluation of cytomorphology. 
Table 1. Characteristics of the esophageal cancer of the studied patients

\begin{tabular}{|c|c|c|c|c|c|c|c|c|c|}
\hline & $\begin{array}{l}\text { Patients } \\
\text { (n) }\end{array}$ & $\begin{array}{c}\text { with } \\
\text { CTCs }\end{array}$ & $(\%)$ & $\begin{array}{l}\text { AdenoCa } \\
\text { (n) }\end{array}$ & $\begin{array}{l}\text { with } \\
\text { CTCs }\end{array}$ & $(\%)$ & $\operatorname{SCC}(n)$ & $\begin{array}{l}\text { with } \\
\text { CTCs }\end{array}$ & $\%$ \\
\hline Total & 43 & 27 & 62.8 & 20 & 15 & 75 & 23 & 12 & 52.2 \\
\hline \multicolumn{10}{|l|}{ T stage } \\
\hline $\mathrm{T} 1$ & 1 & 0 & $\mathbf{0}$ & 0 & 0 & $\mathbf{0}$ & 1 & 0 & $\mathbf{0}$ \\
\hline $\mathrm{T} 2$ & 3 & 3 & 100 & 1 & 1 & 100 & 2 & 2 & 100 \\
\hline $\mathrm{T} 3$ & 23 & 13 & 56.5 & 16 & 11 & 68.8 & 7 & 2 & 28.6 \\
\hline $\mathrm{T} 4$ & 16 & 10 & 62.5 & 3 & 2 & 66.7 & 13 & 8 & 61.5 \\
\hline \multicolumn{10}{|l|}{ N stage } \\
\hline No & 10 & 7 & 70 & 4 & 3 & 75 & 6 & 4 & 66.7 \\
\hline $\mathrm{N} 1$ & 11 & 5 & 45.5 & 7 & 5 & 71.4 & 4 & 0 & $\mathbf{0}$ \\
\hline $\mathrm{N} 2$ & 9 & 6 & 66.7 & 5 & 4 & 80 & 4 & 2 & 0.5 \\
\hline N3 & 13 & 9 & 69.2 & 4 & 3 & 75 & 9 & 6 & 66.7 \\
\hline \multicolumn{10}{|l|}{ M stage } \\
\hline M0 & 35 & 24 & 68.6 & 18 & 14 & 77.8 & 17 & 10 & 58.8 \\
\hline M1 & 8 & 3 & 37.5 & 2 & 1 & $\mathbf{5 0}$ & 6 & 2 & 33.3 \\
\hline \multicolumn{10}{|l|}{ Disease stadium } \\
\hline I & 4 & 3 & 75 & 1 & 1 & 100 & 3 & 2 & 66.7 \\
\hline II & 5 & 3 & 60 & 3 & 2 & 66.7 & 2 & 1 & 50 \\
\hline III & 26 & 18 & 69.2 & 14 & 11 & 78.6 & 12 & 7 & 58.3 \\
\hline IV & 8 & 3 & 37.5 & 2 & 1 & 50 & 6 & 2 & 33.3 \\
\hline \multicolumn{10}{|l|}{ Others } \\
\hline Resectable & 26 & 18 & 69.2 & 14 & 11 & 78.6 & 12 & 7 & 58.3 \\
\hline Non-resectable & 17 & 7 & 41.9 & 6 & 2 & 33.3 & 11 & 5 & 45.4 \\
\hline Neoadjuvant therapy & 18 & 10 & 55.6 & 15 & 10 & 83.3 & 3 & 0 & $\mathbf{0}$ \\
\hline
\end{tabular}

AdenoCa - adenocarcinoma; CTCs — circulating tumor cells; SCC — squamous cell carcinoma

Cells captured on the separating membrane (single cells or cells in clusters) were identified, the photographs were digitized, and evaluated by a trained researcher and/or experienced pathologist. Cells presenting characteristics listed below were defined as CTCs due to the presence of the following criteria: (a) nuclear size equal or larger than $10 \mu \mathrm{m}$; (b) presence of a visible cytoplasm; (c) prominent nucleoli (d) high nuclear-cytoplasmic ratio, which was not necessarily true in case of in vitro cultured cells (e) irregular nuclear contour.

\section{Results}

We report here successful CTCs detection in the blood of EC patients (Figures 1-3). Table 1 shows that the CTCs were detected in $27 / 43$ patients $(62.8 \%)$. Detection of CTCs was higher in patients with adenocarcinoma $(75 \%)$ than squamous cell tumor $(52 \%)$, and this might be caused by the disproportion in the numbers of patients in different stadium of the disease in the adenocarcinoma and squamous cell carcinoma subgroups.

The overall size-based filtration approach enabled to isolate viable CTCs (Figure 2). The general characteristics of the captured CTCs is a relatively big nucleus (more than $10 \mu \mathrm{m}$, Figure 1), which shows prominent nucleoli (4 and more) reflecting the transcriptional activity of the cells. This feature reflects the potential of CTCs to survive under the in vitro conditions. We are able to grow the EC CTCs maximally for 14 days. We could not obtain confluent cultures of these cells; however, we observed two types of subcultures during the cultivation process. The first one was composed of cells which grew on the membrane (Figure 1) while the second one consisted of cells which were growing through the pores in the membrane and formed colonies on the bottom of a culture well.

Culturing of the esophageal cancer CTCs in vitro enabled to have more biological material to further characteristics such as immunohistochemical analysis: we 

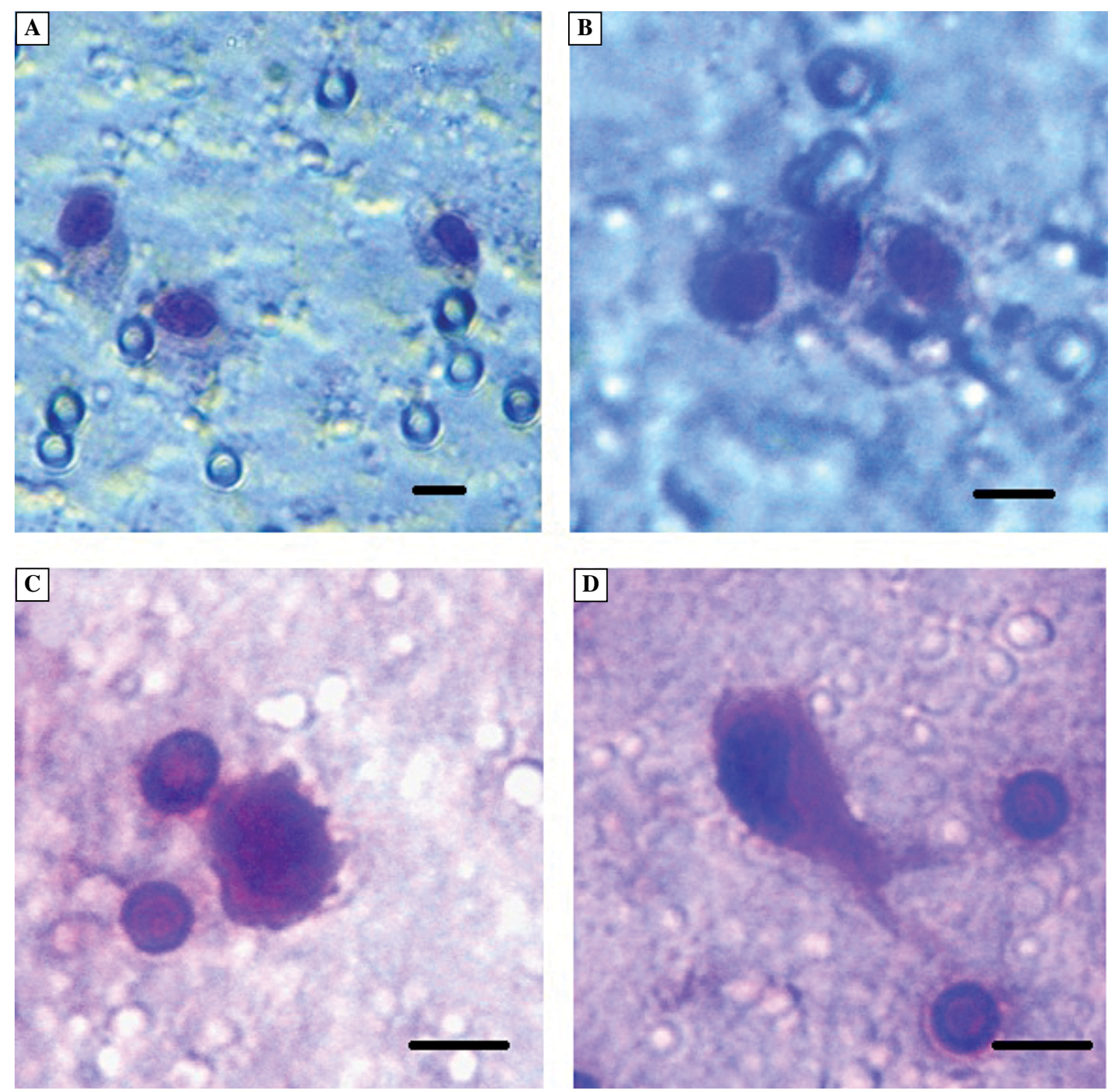

Figure 1. Circulating tumor cells (CTCs) captured on the porous membrane filter, stained by May-Grünwald method. A and B. CTCs after 1 day in culture. C and D. CTCs cultured for 3 days in vitro. Scale bar $10 \mu \mathrm{m}$

used this method to detect in the CTCs cytokeratin 18 to prove their epithelial (gastroepithelial) origin (Figure 3).

We do not have recently the survival analysis of the tested patients group, but thanks to the obtained data, we were able to characterize the CTCs dissemination in the pre-defined patient subgroups (Table 1). Interestingly patients with resectable EC were in $69.2 \%$ cases CTCs-positive, while in the group with non-resectable EC only $42.9 \%$ patients showed CTCs positivity. This might have been caused by the fact, that some of the non-resectable patients were undergoing the neoadjuvant chemotherapy resulting in the decrease of CTCs in the blood. Unfortunately, we do not have all data concerning neoadjuvancy yet. So far the neoadjuvant therapy was administered to 18 patients. From these 18 patients, there CTCs were detected in $10(55.5 \%)$. All these patients have been undergoing only chemotherapy treatment. This in general confirms a very advanced stage of the disease, as could be expected if neoadjuvant therapy is planned.
Nevertheless the CTCs-positivity reflects the disease stadium as well as lymph node involvement. Moreover, there are more patients affected by the spread of the CTCs in stage III than in stage IV of the disease, which may be caused by the small number of the patients in this subgroup indicated to primary tumor resection.

\section{Discussion}

In the presented study we report the CTCs detection in the blood of EC patients and their subsequent in vitro culture. The study was not designed to reflect the survival analysis, but to answer the question, if it could be possible to isolate viable EC-CTCs which could be cultured in vitro. The data on the CTCs presence could be than further used for patient stratification in case of neoadjuvant treatment and surgery. The in vitro cultures can be also a source of biological material for further downstream analysis. 

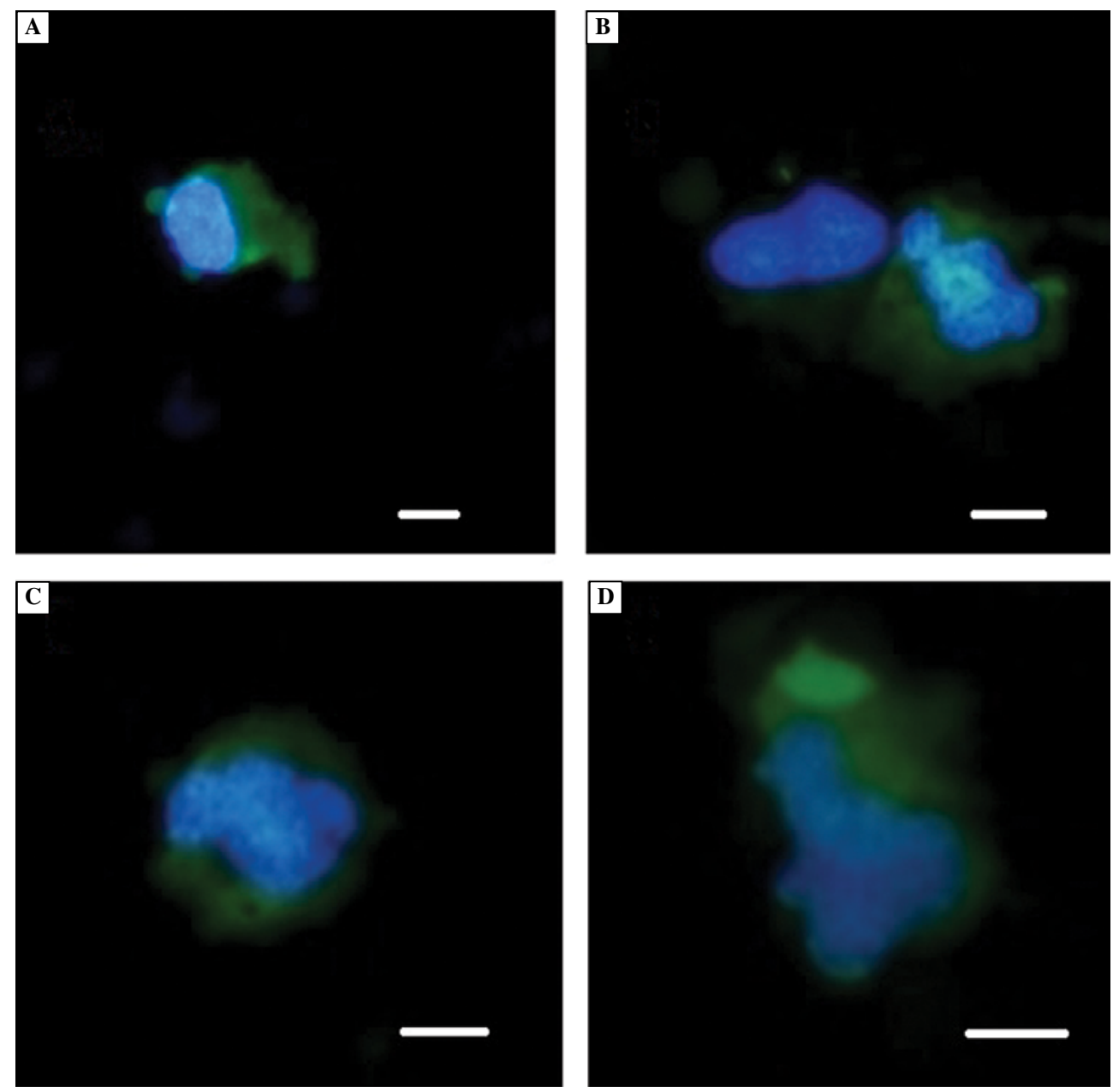

Figure 2. CTCs captured on the membrane filter visualized by blue vital nuclear stain (NucBlue ${ }^{\mathrm{TM}}$ ) and green vital cytoplasmic stain $\left(\right.$ CellTracker $\left.{ }^{\mathrm{TM}}\right)$. Scale bar $10 \mu \mathrm{m}$. Big nuclei with irregular contours were observed very often

The detection of CTCs in blood requires highly sensitive, specific, and reproducible methods. Various techniques are available to isolate CTCs from blood by exploiting their unique properties, including tissue specific nucleic sequences, cell surface markers and physical properties. Investigators have used different blood collection volumes, sample processing methods and criteria to define CTCs [9]. Antibody-based immuno-identification has been the mainstream for CTCs detection. This strategy, however, is limited by the availability and specificity of the antibodies.

In the past detection of CTCs from peripheral blood (PB) of EC patients by quantitative PCR (qPCR) has been reported as a very promising using several genes. Liu et al. aimed at establishing a quantitative system for evaluating the role of CTCs in blood from patients who underwent surgical resection in the treatment of EC [10]. One hundred fifty-five PB samples from 53 EC patients were collected before the surgery (B-1), immediately after surgery (B0), and on the

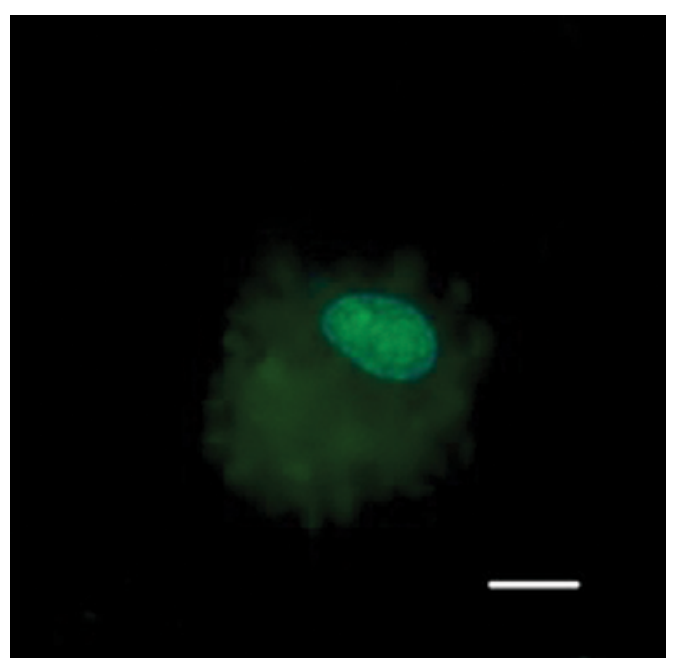

Figure 3. CTCs captured on the membrane filter. The cell origin has been visualized by anti-CK18 antibody (FITC-labelled). The nucleus was visualized by DAPI-staining. Scale bar $10 \mu \mathrm{m}$ 
$3^{\text {rd }}$ day postoperatively $(\mathrm{B}+3)$. A qPCR method based on carcinoembryonic antigen (CEA) mRNA gene expression was designed for the detection of CTCs. The authors showed a significant difference between $\mathrm{B}-1$ and $\mathrm{B} 0(\mathrm{p}=0.0001)$ and between $\mathrm{B}-1$ and $\mathrm{B}+3$ $(p=0.0209)$. Fifty percent of patients with $r>0.4$ $(\mathrm{r}=\mathrm{CTC}$ ratio of $\mathrm{B}+3$ over $\mathrm{B} 0)$ showed tumor recurrence within 1 year after surgery, whereas the probability was only $14.3 \%$ for patients with $r<0.4$ $(\mathrm{p}=0.043)$. This report indicates a prognostic utility of CTCs evaluation in EC.

Furthermore, qPCR of tumor-specific mRNA is characterized by higher sensitivity in comparison to protein-based methods and usually ranges within a detection range of 1 to 10 tumor cells among $10^{6}-10^{7}$ blood mononuclear cells [11]. Nevertheless, the high sensitivity of qPCR is problematic when false-positive results are encountered, as it is the case with sample contamination (genomic DNA versus cDNA) and illegitimate transcription (low-level nonspecific transcription of certain genes) [11].

In recent years, a great number of molecular markers have been identified in the pathogenesis of EC which could be used as potential predictive and prognostic markers. These markers are mainly associated with the six hallmarks of cancer: self-sufficiency in growth signals; insensitivity to antigrowth signals; avoidance of apoptosis; limitless replicative potential; sustained angiogenesis; and tissue invasion and metastasis [12].

Tumor markers such as cytokeratin 19, cytokeratin 20, squamous cell carcinoma antigen (SCC-Ag), deltaNp63, and CEA have all been used with varying degrees of sensitivity and specificity $[9,13-18]$. However, these studies have also revealed a major problem in that some of these tumor markers are expressed in normal blood cells and frequently in normal epithelial cells as well. This inevitably leads to false-positive results that greatly diminish the correlation between tumor markers and some of the well-known clinical and pathological prognostic factors.

To enhance the prediction power of mRNA marker, a combination of markers should be tested. As the amount of CTCs material is limited, the in vitro culture of CTCs, such as demonstrated in our study, could help to overcome these problems.

Other studies provide evidence that some tumor cells may undergo full or partial epithelial-to-mesenchymal transition (EMT) during the process of invasion and intravasation. Likewise, cells may undergo mesenchymal-to-epithelial transition after EMT to re-express some markers that were previously lost [19, 20]. Cells that have undergone EMT are associated with a stem cell phenotype and may resist apoptotic signals, making them more likely to survive in circulation and create deposits of therapeutically resistant metastasis [21].

Additionally, during the enrichment process by capturing CTCs there is a significant loss of CTCs; this loss could be of great importance given the fact that tumor cells represent less than $0.1 \%$ of the enriched population [22]. In general there are conflicting results concerning the superiority of immunomagnetic enrichment over traditional methods: although considered to provide greater specificity, immunomagnetic enrichment has been shown to be hampered by poor sensitivity [23].

Nagrath et al. demonstrated that the average CTCs number per $\mathrm{mL}$ of whole blood was approximately only 79-155 in various cancers [24]. Similarly, in contrast to the FDA-cleared CellSearch epithelial markers-based method [25], an investigative 'CellSearch profile approach' could detect an approximately 30 -folds higher number of the median CTCs counts by using the same paired blood samples in breast cancer [26]. These findings indicate that the actual CTCs numbers in patients' blood could be at least 30-100 times higher than that currently reported by the only FDA-cleared CellSearch system.

Size-based isolation techniques of CTCs have the advantage to be independent of antibody availability. We used MetaCell ${ }^{\circledR}$ protocol with pre-defined pores filter which enables quick isolation of CTCs from blood without affecting the cells by interaction with detecting antibody or lysing solution. We proved that these CTCs are then suitable for subsequent culturing. The CTCs are separated independently on the heterogeneity or pleomorphism of CTCs. The CTCs enriched from blood on the separating membrane are labeled as 'virgin CTCs' since these are the most similar to metastatic tumor present in circulation. These cells are suitable to be cultured and perform subsequent molecular testing with regard to monitoring of chemotherapy efficiency.

Changes in the counts of CTCs appear to reflect more rapidly the impact of the anti-cancer treatment, which can be otherwise reported in as little as a few weeks. Beyond an in vitro number count, an ex vivo functional study on patient-derived CTCs might support an immediate treatment decision regarding surgery eligibility. To reveal the real-time, individualized drug-target interaction, viable CTCs, immediately enriched from cancer patient's blood, can be also used to target new anti-cancer drug candidates. 


\section{Acknowledgments}

This study was supported by Grant provided by the League Against Cancer Prague, Czech Republic (Grant with title: Sledování hladiny CTC u vybraných nádorů gastrointestinálního traktu) and grant of the Czech Ministry of Health: IGA NT14439-3/2013, and also supported by the research project P27/2012 awarded by the Charles University in Prague.

\section{References}

1. Jemal A, Siegel R, Ward E et al. Cancer statistics, 2007. $C A$ Cancer J Clin. 2007;57:43-66.

2. Enzinger PC, Mayer RJ. Esophageal cancer. $N$ Engl J Med. 2003;349:2241-2252.

3. Katayama A, Mafune K, Tanaka Y et al. Autopsy findings in patients after curative esophagectomy for esophageal carcinoma. J Am Coll Surg. 2003;196:866-873.

4. Elshimali YI, Grody WW. The clinical significance of circulating tumor cells in the peripheral blood. Diagn Molec Pathol. 2006;15:187-194.

5. Matsusaka S, Chìn K, Ogura M et al. Circulating tumor cells as a surrogate marker for determining response to chemotherapy in patients with advanced gastric cancer. Cancer Sci. 2010;101:1067-1071.

6. Steinert G, Schölch S, Koch M, Weitz J. Biology and significance of circulating and disseminated tumour cells in colorectal cancer. Langenbecks Arch Surg. 2012;397:535-542.

7. Doyen $\mathrm{J}$, Alix-Panabières $\mathrm{C}$, Hofman $\mathrm{P}$ et al. Circulating tumor cells in prostate cancer: a potential surrogate marker of survival. Crit Rev Oncol Hematol. 2012;81:241-256.

8. Serrano MJ, Rovira PS, Martínez-Zubiaurre I, Rodriguez MD, Fernández M, Lorente JA. Dynamics of circulating tumor cells in early breast cancer under neoadjuvant therapy. $\operatorname{Exp}$ Ther Med. 2012;4:43-48.

9. Eliasova P, Kolostova K, Kobierzycki C, Bobek V. Clinical studies monitoring circulating and disseminated tumor cells in gastrointestinal cancers. Folia Histochem Cytobiol. 2013;51:265-277.

10. Liu Z, Jiang M, Zhao J, Ju H. Circulating tumor cells in perioperative esophageal cancer patients: quantitative assay system and potential clinical utility. Clin Cancer Res. 2007;10: 2992-2997.

11. Lurje G, Schiesser M, Claudius A, Schneider PM. Circulating tumor cells in gastrointestinal malignancies: current techniques and clinical implications. J Oncol. 2010;2010:392-652.

12. Vallböhmer D, Brabender J, Metzger R, Hölscher AH. Genetics in the pathogenesis of esophageal cancer: possi- ble predictive and prognostic factors. $J$ Gastrointest Surg. 2010;14(Suppl 1):75-80.

13. Huang P, Wang J, Guo Y et al. Molecular detection of disseminated tumor cells in the peripheral blood in patients with gastrointestinal cancer. J Cancer Res Clin Oncol. 2003;129:192-198.

14. Ikoma D, Ichikawa D, Ueda Y et al. Circulating tumor cells and aberrant methylation as tumor markers in patients with esophageal cancer. Anticancer Res. 2007;27:535-539.

15. Ito $H$, Kanda $T$, Nishimaki $T$ et al. Detection and quantification of circulating tumor cells in patients with esophageal cancer by real-time polymerase chain reaction. J Exp Clin Cancer Res. 2004;23:455-464.

16. Kaganoi J, Shimada Y, Kano M et al. Detection of circulating oesophageal squamous cancer cells in peripheral blood and its impact on prognosis. Br J Surg. 2004;91:1055-1060.

17. Koike M, Hibi K, Kasai Y et al. Molecular detection of circulating esophageal squamous cell cancer cells in the peripheral blood. Clin Cancer Res. 2002;8:2879-2882.

18. Nakashima $S$, Natsugoe $S$, Matsumoto $M$ et al. Clinical significance of circulating tumor cells in blood by molecular detection and tumor markers in esophageal cancer. Surgery. 2003;133:162-169.

19. Tsuji T, Ibaragi S, Hu GF. Epithelial-mesenchymal transition and cell cooperativity in metastasis. Cancer Res. 2009;69:7135.

20. Armstrong AJ, Marengo MS, Oltean S et al. Circulating tumor cells from patients with advanced prostate and breast cancer display both epithelial and mesenchymal markers. Mol Cancer Res. 2011;9:997-1007.

21. Mego M, Mani SA, Cristofanilli M. Molecular mechanisms of metastasis in breast cancer - clinical applications. Nat Rev Clin Oncol. 2010;7:693-701.

22. Morgan TM, Lange PH, Vessella RL. Detection and characterization of circulating and disseminated prostate cancer cells. Front Biosci. 2007;12:3000-3009.

23. Ausch C, Dandachi N, Buxhofer-Ausch V et al. Immunomagnetic CD45 depletion does not improve cytokeratin 20 RT-PCR in colorectal cancer. Clin Chem Lab Med. 2007;45: 351-356.

24. Nagrath S, Sequist LV, Maheswaran S et al. Isolation of rare circulating tumour cells in cancer patients by microchip technology. Nature. 2007;450:1235-1239.

25. Fehm T, Müller V, Aktas B et al. HER2 status of circulating tumor cells in patients with metastatic breast cancer: a prospective, multicenter trial. Breast Cancer Res Treat. 2010;124:403-412.

26. Flores LM, Kindelberger DW, Ligon AH et al. Improving the yield of circulating tumour cells facilitates molecular characterisation and recognition of discordant HER2 amplification in breast cancer. Br J Cancer. 2010;102:1495-1502.

Submitted: 25 March, 2014

Accepted after reviews: 9 September, 2014 